\title{
Efficient Image Compression based on Seam Carving for Arbitrary Resolution Display Devices
}

\author{
E.Salma \\ Student /ME (Communication Systems), \\ G.K.M College of Engineering and Technology, \\ Chennai, Tamil Nadu, India
}

\author{
J.P. Josh Kumar \\ Assistant Professor (Department of ECE) \\ G.K.M College of Engineering and Technology, \\ Chennai, Tamil Nadu, India
}

\begin{abstract}
In mobile communications, image retargeting is generally required at the user end. The existing coding techniques do not support content-aware spatial scalability. The work presented in this paper addresses the increasing demand of compression techniques for arbitrary resolution display devices. This paper throws light on efficient content-aware compression of medical images. The principle of seam carving is incorporated into a wavelet codec. In the meantime, discrete wavelet transform (DWT) is performed and DWT coefficients are grouped. The coefficients are encoded and decoded using SPIHT and the image can be reconstructed in a content-aware manner. Experimental results show that the retargeted image generated by the proposed technique preserve sensitive image content and has been proven efficient for medical images by achieving excellent compression performances.
\end{abstract}

\section{Keywords}

Content-aware, discrete wavelet transform (DWT), image compression, seam carving (SC), spatial scalability

\section{INTRODUCTION}

DURING the past years, image compression has been extensively studied. Among the existing codecs, the most commonly used ones are mainly based upon discrete wavelet transform (DWT), such as JPEG 2000 and SPIHT. Furthermore, JPEG 2000 with a region of interest (ROI) allows the ROIs of the image to be coded with better quality than that of non-ROIs. However, the spatial scalability is not fully supported in the codecs above, and only dyadic resizing can be achieved without considering the image content (as a result, the distortion of original important visual content may be severe in the retargeted image).Recently, inspired by the image/video inpainting techniques a number of pruning-based coding schemes have been proposed towards visual quality rather than pixel-wise fidelity. Nowadays, as the size of portable devices (e.g., laptops, PDAs, and mobile phones) continue diversifying, the existing coding schemes cannot be directly applied, and an additional image retargeting process (e.g., down sampling, cropping, warping or seam carving (SC) ) is needed in the receiving end. However, for the resource-limited mobile devices, it is not always possible and economical to perform sophisticated content-aware image resizing. Therefore, content-based spatialscalable image compression for arbitrary resolution is becoming one of the emergent challenges for universal access i.e., one can access any information over any network from anywhere through any type of display devices. The first proposed contentaware multisize image compression scheme. The basic idea is that, by using the significance map based upon SC, an image is decomposed into two components: ROI and non-ROI. For the ROI, it is encoded by the SPIHT codec and the size is to be altered; for the non-ROI, the pixels are grouped as a sequence of seams, i.e., a connected path of low-energy pixels crossing the
non-ROI region from top to bottom (a vertical seam), or from left to right (a horizontal seam). The seam information is encoded by adaptive arithmetic coding algorithm and during image decoding with the need of resizing, the seams with low energy are deleted. Experimental results showed that the coding efficiency is far below the wavelet-based SPIHT codec: a 2.68bpp SPIHT-coded image achieved the same peak-signal-tonoise ratio (i.e. PSNR $40.5 \mathrm{~dB}$ ) as a 5.85-bpp seam-coded image. In addition, since the ROI and non-ROI are encoded using different image coding schemes, severe block-artifacts occur on the boundaries of the ROI and non-ROI regions. Following the concepts of various references a content-based spatial-scalable coding framework with concentration and dilution (termed as CD-based image coding) was developed. The principle of this codec is as follows: at the encoder side, the original image is first partitioned into ROI and non-ROI regions, the pixels in the non-ROI are then grouped into seams by an hierarchical search process, the resultant seams are removed that is, for the non-ROI region, no data are encoded and transmitted other than the position information of each seam; the ROI regions are encoded.

The remainder of this paper is organized as follows. Section II reviews the relevant principles and backgrounds. Section III introduces the proposed content-aware spatial-scalable image coding scheme in detail, including block-based seam energy map generation, content-based SPIHT encoding, side information encoding, and spatial scalability. Section IV demonstrates the simulation results compared with other relevant state-of-the-art image coding schemes, with subjective and objective evaluation, while Section V concludes this paper.

\section{BACKGROUND REVIEW} \subsection{Seam Carving Overview}

A seam is defined as an 8-connected path of pixels from either top to bottom (vertical seam) or left to right (horizontal seam). In order to maintain an image's rectangular shape, a seam contains exactly one pixel per row (vertical seam) or per column (horizontal seam). The optimum path is determined by generating a cumulative energy map $M$ and traversing from the bottom edge (or right edge) back to the top edge (or left edge), maintaining a connected path and selecting the minimum value at each row (or column). This path of pixels is the seam and is removed. The process then starts over, continuing until the desired resolution is met. While there are several seam carving operations - vertical seam removal/insertion, horizontal seam removal/insertion, combined vertical and horizontal seam removal/insertion - they are all based on the process of identifying a single seam. Therefore, for the sake of brevity, development of our model will be based on the selection of vertical seams only, but can be easily extended to the other listed operations[5].

The generation of the cumulative energy map $(i, \mathrm{j})$ as defined in [2] and generalized here for vertical seam removal: 


$$
\begin{array}{r}
(i, j)=\min \{( \\
(i-1,-1)+\boldsymbol{D} i, j p R \\
(i-1, \mathrm{j})+\boldsymbol{D} i, j p C \\
(i-1,+1)+\boldsymbol{D} i, j p L\}
\end{array}
$$

\subsection{Lifting Scheme}

The Lifting scheme is a technique for both designing wavelets and performing the discrete wavelet transform. In this scheme the signal is divided like a zipper. The lifting scheme can rightly be called as second generation wavelet transform due to its high execution speed. It has perfect reconstruction and speed up by a factor of 2. The drawback analyzed in spite of its outstanding properties is its poor image quality.

\section{PROPOSED CODEC DESCRIPTION}

In our work, SC is applied to improve the spatial scalability of the conventional wavelet-based SPIHT scheme. The block diagram of the proposed codec is shown in Fig.1, which involves two major aspects: the block-based seam energy map and seam-based SPIHT coding.

\subsection{Discrete Wavelet Transform}

The Wavelet Transform provides a time-frequency representation of the signal. It was developed to overcome the short coming of the Short Time Fourier Transform (STFT), which can also be used to analyze non-stationary signal. The Wavelet Series is just a sampled version of CWT and its computation may consume significant amount of time and resources, depending on the resolution required. The Discrete Wavelet Transform (DWT), which is based on sub-band coding is found to yield a fast computation of Wavelet Transform. It is easy to implement and reduces the computation time and resources required. There is a wide range of applications for Wavelet Transforms. They are applied in different fields ranging from signal processing to biometrics

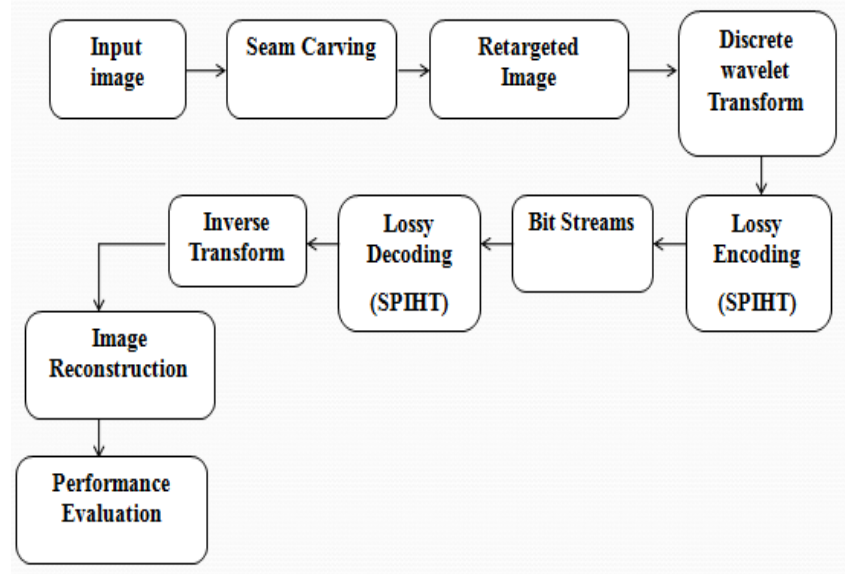

Figure 1: Block diagram of proposed image codec

\subsection{Content-Based SPIHT Coding}

Set partitioning in hierarchical trees (SPIHT) [2] is based on the multiscale 2-D DWT and exploits the self-similarity across scales by using set partitioning.

A seam-guided SPIHT scheme is proposed for the encoding of wavelet coefficients. From Section II-B, it is already known that the DWT coefficients are grouped as spatial orientation trees (SOTs). In the conventional SPIHT scheme, the SOTs are scanned and encoded in a zig-zag order, and the coding process is performed from the MSB to the LSB.

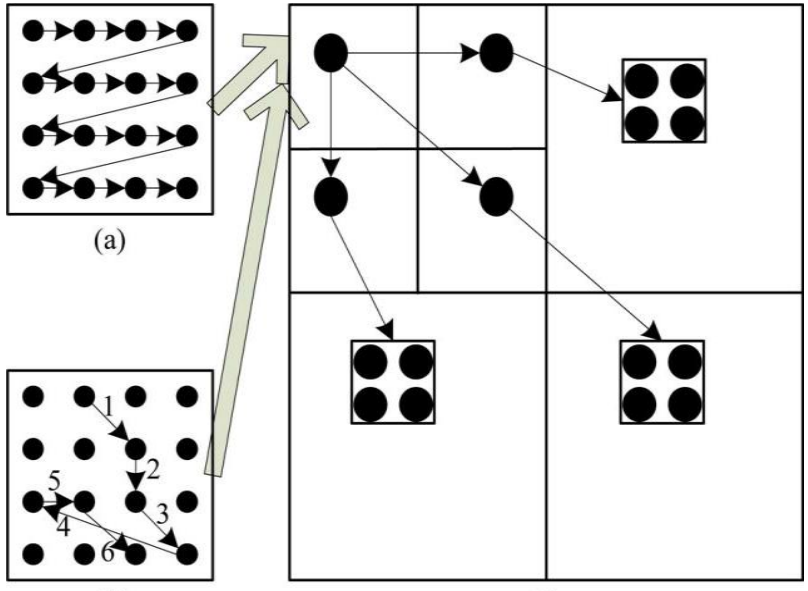

(b)

(c)

Figure2: Wavelet coefficients coding. (a) Conventional scanning order in low frequency subband. (b) Seam energy guided scanning order in the proposed scheme. (c) SOT (two scales being used for illustration purposes here).

\subsection{Spatial Scalability}

The SPIHT-coded bitstream are transmitted in a content-aware manner. By using the received side information and bitstream, the reconstructed image with a different resolution can be generated and then uniform scaling is performed for the ROI regions to obtain the reconstructed image with required size.

\section{SIMULATIONS AND ANALYSIS}

\subsection{Initial Parameters}

The experiments conducted to evaluate the compression performance are reported here. SPIHT is well suited for medical images, portraits and landscapes. Thus the proposed compression technique can be very well explained by the medical(brain) image in Fig 3. The primary image Fig 3(b) is initially detected and later the secondary image (d) is detected after the boundary detection process stated in (c).

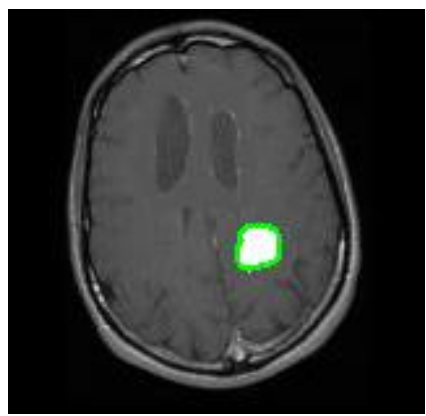

Figure 3: a. Original image

b. Primary image

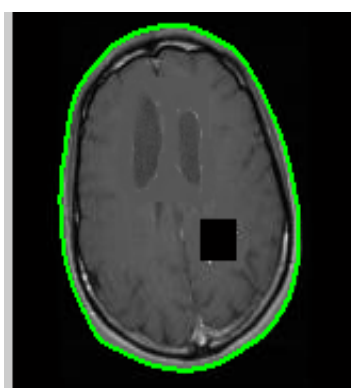

c. Boundary detection

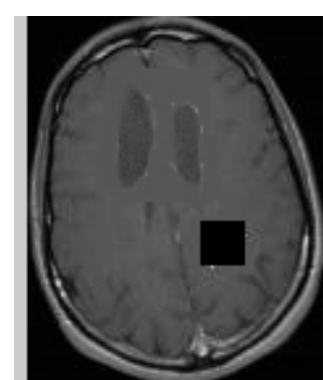

d. Secondary image 


\subsection{DWT based Seam Carving}

The step by step procedure for DWT based Seam Carving is illustrated in Fig 4.
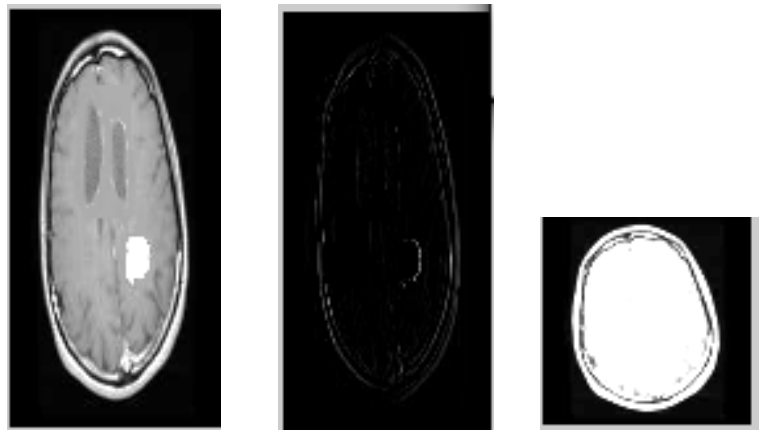

Figure 4: a. Column b. Column Edge Compression Detection d. Row edge detection Horizontal Band

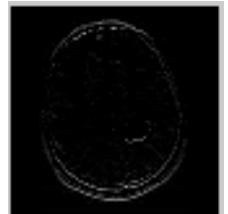

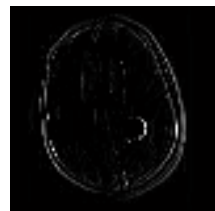

e. Row and Column compression

Vertical Band c. Row \&Column compression Average band

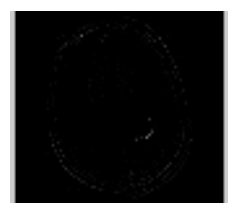

f. Diagonal Band

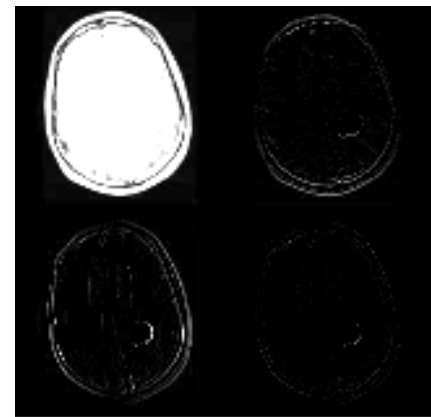

g. Combined image

\subsection{Seam SPIHT Results}

The Seam based SPIHT encoding and decoding is performed for the DWT computed image. The high PSNR value obtained and the processes carried on is listed in TABLE I

TABLE 1. MATLAB analysis result for SPIHT algorithm

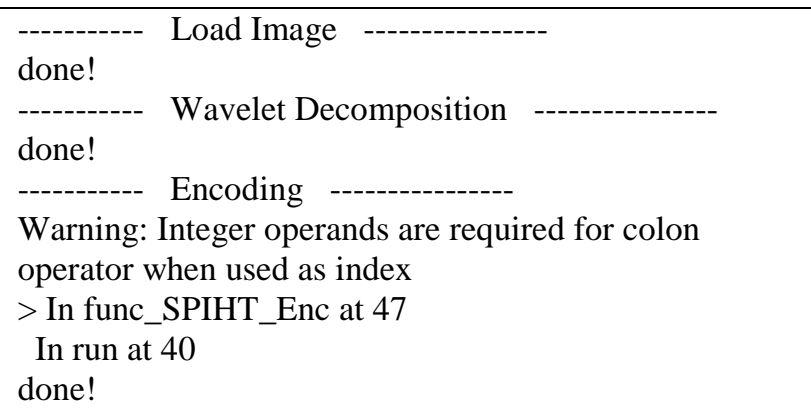

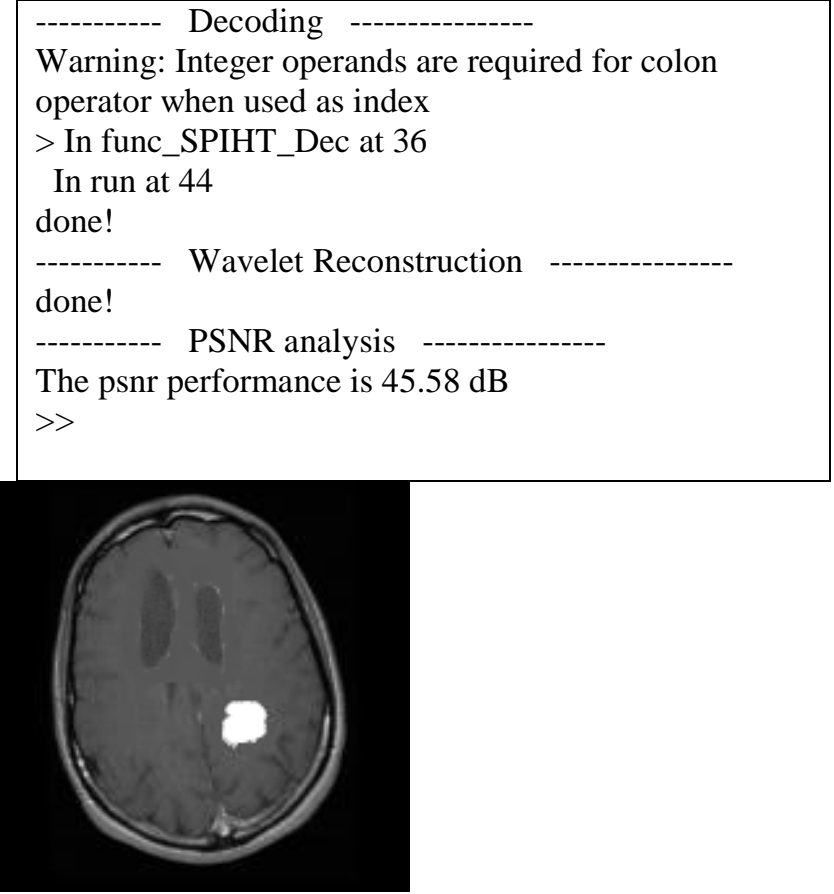

Figure 5: a. original image

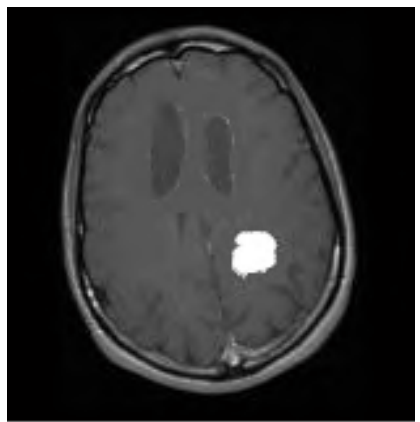

b. Reconstructed image

\subsection{Testing and Analysis}

TABLE 2. Experimental PSNR values for the test images

\begin{tabular}{|c|l|}
\hline Test images & PSNR value \\
\hline Brain & $\mathbf{4 5 . 5 8} \mathbf{~ d B}$ \\
\hline Lena & $\mathbf{4 5 . 4 7} \mathbf{d B}$ \\
\hline
\end{tabular}

\section{CONCLUSION}

This proposed technique has provided a solution for delivering images to diversifying end-user display terminals with arbitrary resolutions, maintaining visually important content as far as possible and without extra computational burden from the decoding perspective. As far as the analysis is carried out Seam SPIHT provides the excellent PSNR performance and image quality in the medical image. the results obtained maintains the essential contents which is required for content aware image resizing. Thus SPIHT which is rightly the state of the art algorithm suitable for medical images is expected to yield 
excellent results in any kind of multimedia images. results . The proposed codec (Seam-SPIHT) has its roots primarily in the DWT-based SPIHT scheme, with different coefficient coding order. Experimental results have shown that the retargeted images generated by the proposed codec preserve important and sensitive image content (i.e., in a content-aware manner).

\section{ACKNOWLEDGMENT}

The authors would like to thank Apollo Hospital for providing brain image samples used in the study. We would like to express thanks to our college Deputy Registrar Dr. K. O. Joseph and Head of the Department Dr. D. Balasubramaniam for their invaluable guidance and support.

\section{REFERENCES}

[1] A. Said and W. A. Pearlman, "A new, fast and efficient image codec based on set partitioning in hierarchical trees," IEEE Trans. Circuits Syst. Video Technol., vol. 6, no. 3, pp. 243-250, Jun. 1996.

[2] C. Christopoulos, J. Askelof, and M. Larsson, "Efficient methods for encoding regions of interest in the upcoming JPEG 2000 still image coding standard," IEEE Signal Process. Lett., vol. 7, no. 9, pp. 247-249,Sep.2000

[3] D. S. Taubman and M. W. Marcellin, JPEG 2000: Image Compression Fundamentals, Standards and Practice. New York: Springer,Nov.2001.

[4]. Information Technology-JPEG 2000 Image Coding System: Core Coding System, IEEE Std. ISO/IEC 15 4441, Sep. 2004.
[5] S. Avidan and A. Shamir, "A seam carving for contentaware image resizing," ACM Trans. Graphics, vol. 26, no. 3, pp. 10-19, Jul.2007.

[6] D. Liu, X. Y. Sun, F.Wu, S. P. Li, and Y. Q. Zhang, "Image compression with edge-based inpainting," IEEE Trans. Image Process., vol. 17, no. 10, pp. 1273-1287, Oct. 2007.

[7] N. T. N. Anh, W. X. Yang, and J. F. Cai, "Seam carving extension: A compression perspective," in Proc. ACM Conf. Multimedia,Oct.2009,pp.825-828

[8] D. T. Vo, J. Sole, P. Yin, C. Gomila, and T. Q. Nguyen, "Selective data pruning-based compression using highorder edge-directed interpolation," IEEE Trans. Image Process., vol. 19, no. 2, pp.399-409,Feb.2010.

[9] Y. Tanaka,M. Hasegawa,"Improved image concentration for artifact-free image dilution and its application to image coding," in Proc. IEEE Int. Conf. Image Process., Sep. 2010, pp. 1225-1228

[10] M. Rubinstein, D. Gutierrez, O. Sorkine, and A. Shamir, "A comparative study of image retargeting," ACM Trans. Graphics, vol. 29, no. 6, pp. 160-168, Dec. 2010.

[11] Y. J. Liu, Y.M. Xuan, W. F. Chen, and X. L. Fu, "Image retargeting quality assessment," in Proc. EUROGRAPHICS, Apr. 2011, vol. 30, no. 2, pp. 583-592.

[12] B. Manjunath, O. J. Vasudevan, and A. Yamada, "Color and texture descriptors," IEEE Trans. Circuits Syst. Video Technol., vol. 11, no. 6,pp. 703-715, Jun. 2001. 